\title{
Changes in NfL Plasma Levels and Association with Cognitive and Imaging Markers of Alzheimer's Disease
}

Sumali Bajaj ( $\nabla$ sumali.bajaj@seh.ox.ac.uk)

University of Oxford

Christoforos Hadjichrysanthou

Imperial College London

Kevin McRae-McKee

Imperial College London

Frank De Wolf

Imperial College London

Roy M. Anderson

Imperial College London

\section{Research Article}

Keywords: NfL plasma levels, cognitive and imaging markers, plasma neurofilament light chain ( $\mathrm{pNfL}$ ), Alzheimer's disease (AD)

Posted Date: July 1st, 2021

DOI: https://doi.org/10.21203/rs.3.rs-658952/v1

License: (1) This work is licensed under a Creative Commons Attribution 4.0 International License. Read Full License 


\section{Abstract}

INTRODUCTION: We assessed the association of plasma neurofilament light chain ( $\mathrm{pNfL}$ ) with cognitive decline and neuroimaging markers, and investigated its potential relationship with the clinical progression to dementia due to Alzheimer's disease (AD).

METHODS: Individuals had evidence of amyloid beta accumulation. Linear and beta-regression models were developed to consider: i) the association between $\mathrm{pNfL}$ and cognition, ii) the association between the rate of change (ROC) of $\mathrm{pNfL}$ and that of imaging markers, iii) the temporal dynamics of $\mathrm{pNfL}$ before and after cognitive impairment as assessed by the Clinical Dementia Rating.

RESULTS: Higher levels of pNfL were associated with declining cognition. The ROC of pNfL was associated with the ROC of ventricular, hippocampal and whole brain volumes, but not with PET amyloid. pNfL levels did not reflect the clinical progression of $A D$.

DISCUSSION: pNfL is associated with cognitive decline and brain imaging markers. However, it is not specific to $A D$ clinical diagnosis.

\section{Introduction}

Alzheimer's disease (AD) is diagnosed post mortem ${ }^{1}$ based on the presence of two cardinal pathologies neurofibrillary tangles (Tau) and senile amyloid-beta (Ab) plaques, or in-vivo by abnormal biomarkers of $\mathrm{Ab}$ and pathological Tau ${ }^{2}$. These pathological changes are believed to lead to cognitive and functional impairment. Various biomarkers based on cerebrospinal fluid (CSF), and imaging using structural magnetic resonance imaging (MRI) or positron emission tomography (PET) of amyloid deposition and cerebral glucose metabolism, are used as proxies for observing the underlying neuropathological process in-vivo ${ }^{3}$. However, CSF, MRI and PET biomarkers can be highly invasive, expensive and limited in their availability in many care settings. Therefore, identifying and validating blood-based biomarkers which can help characterise AD progression are a focus of much research at present. There have been recent advances in identifying the validity of blood-based markers, including $A \beta$, Tau and Neurofilament light chain (NfL) $(4,5,6,7)$.

$\mathrm{NfL}$ has been shown to be a promising biomarker for neurodegeneration in various diseases, such as Parkinson's Disease, Frontotemporal Dementia, Amyotrophic Lateral Sclerosis, Huntington's Disease, Multiple Sclerosis and Alzheimer's Disease ${ }^{8,9},{ }^{10}$. NfL is a structural protein which leaks into the cerebrospinal fluid and subsequently into peripheral blood, when there is axonal neuronal damage in the brain. Elevated CSF NfL levels but not total-tau, phosphorylated-tau, or neurogranin levels are shown to be associated with a higher risk of mild cognitive impairment $(\mathrm{MCl}){ }^{11}$. Higher plasma $\mathrm{NfL}(\mathrm{pNfL})$ levels have been shown to be associated with a higher risk of developing AD dementia ${ }^{12}$. Studies have also shown that $\mathrm{pNfL}$ levels are elevated for $A D$ patients and increase over time. The increase in levels of $\mathrm{pNfL}$ over 
time is associated with changes in markers of AD progression, including CSF biomarkers, imaging measures and cognition 6,7 .

In this paper, we build on previous research by developing robust statistical models to consider changes over time in levels of pNfL and established $A D$ markers in individuals at risk of developing non-familial (sporadic) AD. Our aim is to improve understanding of the role of $\mathrm{pNfL}$ as a biomarker for AD. We investigate the effect of baseline and longitudinal pNfL levels on the rate of decline in cognition in individuals that have elevated levels of amyloid in the brain (PET florbetapir SUVR > 1.11 and/or CSF A $\beta_{1}$.

${ }_{42}<880 \mathrm{pg} / \mathrm{mL}$ ) at baseline. We also study the associations between the rate of change (ROC) in $\mathrm{PNfL}$ over time and ROC of other known markers associated with AD clinical progression (cognition, brain structure imaging) in this population. In addition, using the ROC in pNfL levels, we describe the temporal dynamics of $\mathrm{pNfL}$ before and after the onset of symptomatic cognitive impairment (CDR-Sum of Boxes > 0 ) and consider the potential relationship with the clinical progression from the cognitively unimpaired state, to $\mathrm{MCl}$, and finally to $\mathrm{AD}$ dementia.

\section{Methods}

\subsection{Dataset used}

Data used in the analyses reported in this article were obtained from the Alzheimer's Disease Neuroimaging Initiative (ADNI) database (adni.loni.usc.edu). The ADNI was launched in 2003 as a publicprivate partnership, led by Principal Investigator Michael W. Weiner, MD. The primary goal of ADNI has been to test whether serial MRI, PET, other biological markers, and clinical and neuropsychological assessment can be combined to measure the progression of $\mathrm{MCl}$ and early $\mathrm{AD}$.

The ADNI, a longitudinal study which started as a five-year initiative (ADNI-1), has been followed by ADNI Grand Opportunities (ADNI-GO), ADNI-2 and ADNI-3 protocols. The participants, aged 55-90 years, have been recruited at 57 sites in the United States and Canada. The study procedures were approved by the institutional review boards of all participating centers and informed written consent has been provided by all participants.

\subsubsection{Sample selection}

ADNI data were downloaded on 2018/09/28. 797 of 2046 individuals in the database (39\%) had elevated baseline levels of amyloid in the brain (PET florbetapir SUVR $>1.11^{13}$ and/or CSF A $\beta_{1-42}<880 \mathrm{pg} / \mathrm{mL}^{14}$ ). Data from these individuals has been used in the present study (subsequently referred to as 'amyloid positive' individuals). The longitudinal assessment of cognition was divided into two sub-analyses: i) using baseline pNfl levels, and ii) using baseline ROC calculated utilising longitudinal measures of pNfL within individuals. The first sub-analysis included 735 amyloid positive participants (sample A as shown in Figure 1) with baseline measurement of $\mathrm{pNfL}$ and the following longitudinal cognitive assessments: 
Mini-Mental State Examination, MMSE ${ }^{15}$; Alzheimer's Disease Assessment Scale-Cognitive 13, ADAS-Cog $13^{16}$; Clinical Dementia Rating-Sum of Boxes, CDR-SB 17,18; Alzheimer's Disease Composite Score, ADCOMS ${ }^{19}$; Preclinical Alzheimer's Cognitive Composite including Trail-Making Test B, PACC ${ }^{20,21}$. In addition, all individuals had complete data on time (in years) since baseline (e.g. joining the study cohort), age at baseline (years), sex, years of education, and apolipoprotein E (APOE) $₫ 4$ allele binary status (APOE+ defined as having at least one allele). The second sub-analysis included 336 amyloid positive participants (sample B in Figure 1) with two or more measurements of pNfL within 48 months from baseline, repeated measures on the cognitive scores after 48 months since baseline and covariates mentioned above. The dataset used to compare the ROC of $\mathrm{pNfL}$ and the ROC of various imaging markers of $A D$ included 236 amyloid-positive participants (sample $C$ in Figure 1) with two or more measurements of $\mathrm{pNfL}(\mathrm{pg} / \mathrm{mL})$, ventricular volume $\left(\mathrm{mm}^{3}\right)$, hippocampus volume $\left(\mathrm{mm}^{3}\right)$, whole brain volume $\left(\mathrm{mm}^{3}\right)^{22}$, and amyloid accumulation (Standard Uptake Value Ratio SUVr) using Florbetapir in PET scans (AV-45-PET) ${ }^{23}$ within 48 months since baseline to make the analysis comparable and within a relatively short time period so that assumptions of constant rate of change between time points are more robust. All volumetric measures were adjusted for total intracranial volume ${ }^{24}$. For the development of the pNfL trajectory, we utilised data from 501 individuals (sample D in Figure 1) who had two or more measurements of pNfL, ADAS-Cog13 and MMSE (see Figure 1).

\subsection{Statistical Analysis}

\subsubsection{Association between pNFL and longitudinal cognitive decline (Sample A and B)}

We analysed the association between cognitive scores over time (outcome) and continuous baseline pNfL (log10 transformed) using linear mixed effect (LME) models and beta-regression, with random intercepts and slopes to account for repeated outcome measures. Cognitive scores are generally bounded between two numbers, therefore assuming that the errors follow a normal distribution is not appropriate. Beta regression overcomes this by assuming that the errors follow a beta distribution, which can take various shapes depending on the values of its two parameters ${ }^{25}$. Both the LME and beta-regression models were adjusted for time (years) since baseline and the following potential confounders: baseline age (years), sex (binary classification), education level (years), and APOE $₫ 4$ allele status (binary). A quadratic term for time which improved the model fits (as measured by significance of additional term) was included in the linear mixed effects model. Baseline $\mathrm{pNfL}$ values were also grouped into quartiles for visualisation. These were then used to plot the cognitive trajectories corresponding to the baseline quartiles.

Using data collected between baseline and month 48 , we also calculated the average ROC in the pNfL measurement using only the first three visits at which pNfL was measured; we define this ROC as the 
'baseline ROC'. The method used for calculating an individual's ROC is explained in the following paragraph. The last visit used to calculate the baseline ROC was then considered as the new baseline for the subsequent visits at which cognitive scores were measured. In other words, cognitive data from the period used to calculate the baseline ROC was discarded and subsequent analysis were based purely on longitudinal cognitive data recorded after the baseline ROC period. We then tested the hypothesis - is this baseline ROC of pNfL associated with longitudinal cognitive decline - using linear and beta mixed effects models in the same way as explained above, adjusting for the same covariates. Corresponding plots using quartiles of baseline ROC were created.

\subsubsection{Rate of change over time in pNfL and AD-related biomarkers (Sample C)}

In order to estimate the ROC of pNfL for each individual in the cohort, we employed log10 transformed $\mathrm{pNfL}$ values (scaled to have zero mean and unit standard deviation) as the outcome and years since baseline as the predictor. Random intercepts and random slopes were included to allow each individual's trajectory over time to deviate from the overall trend. The individual-specific posterior estimates of the random slopes were used as the measure of the ROC in pNfL over time. The same method was used to calculate the ROC in the imaging markers - ventricular volume, hippocampus volume, whole brain volume, Florbetapir PET SUVr. We then performed linear regression between the estimated ROC in PNfL and ROC in these volumetric brain structure markers and Florbetapir PET SUVr, accounting for the following timeinvariant covariates: baseline age, sex, years of education, and APOE $₫ 4$ allele binary status.

\subsubsection{Temporal dynamics of pNfL and other markers (Sample D)}

For the development of biomarker trajectories, we used a method based on differential-equations described in previous studies ${ }^{26}$. We made two main assumptions: 1 ) all individuals will follow the same general pattern of biomarker trajectory during the progression of $A D$, and 2) the biomarker levels either increase or decrease monotonically with time. For each individual rate of change in the level of a biomarker, as estimated in Section 'Rate of change over time in PNfL and AD-related biomarkers (Sample C)', we first calculated the corresponding mean biomarker level (mean of the biomarker values used for the calculation of the rate of change). A quadratic function was fitted to the data to describe the ROC as a function of the mean. The function that described best the relationship between ROC and mean values was then integrated to produce the average long-term temporal dynamics of the biomarker. For the integration, we assumed that at time zero the mean value of the biomarker is equal to the average value at the point where the CDR-SB score becomes bigger than zero in the data, i.e. first symptomatic cognitive impairment as assessed by CDR-SB is observed ${ }^{27}$. Hence, without loss of generality, we enabled a function transformation so that the biomarker change is described as a function of time from the first 
non-zero CDR-SB score. Using the non-linear least squares method, the individual biomarker values were then synchronised to match the population level trajectory.

Data formatting and plotting was performed in $\mathrm{R}$ version 4.0.3 and $\mathrm{R}$ studio version 1.3.1093. The posterior distributions of the parameters and $95 \%$ credible intervals (Crl) were estimated using Hamiltonian Monte Carlo (HMC) through the rstan interface 2.21.0 ${ }^{28}$ which uses the "No-U-TurnSampler". The Gelman-Rubin statistic Rhat $<1.1$ was used as an indicator for convergence of parameter chains. To infer the model parameters, we report the posterior parameter mean estimates and their respective $95 \%$ Crls. The temporal dynamics of biomarkers as a function of time from first CDR-SB>0 have been estimated in MATLAB R2019a.

\section{Results}

\subsection{Sample Characteristics}

Demographics and biomarker characteristics of our study population at baseline for the four samples used in the analyses (Sample A-D) are given in Table 1. Details of the number of individuals in each analysis are presented in Figure 1.

Table 1

Participant characteristics in different samples. 


\begin{tabular}{|c|c|c|c|c|}
\hline & $\begin{array}{l}\text { Sample A } \\
(\mathrm{n}=735)\end{array}$ & $\begin{array}{l}\text { Sample B } \\
(n=336)\end{array}$ & $\begin{array}{l}\text { Sample C } \\
(n=263)\end{array}$ & $\begin{array}{l}\text { Sample D } \\
(n=501)\end{array}$ \\
\hline Age (years) & $73.78(7.13)$ & $74.61(7.14)$ & $72.56(6.96)$ & $73.31(6.95)$ \\
\hline Sex (female) & $334(45.44 \%)$ & $149(44.35 \%)$ & $120(45.63 \%)$ & $226(45.11 \%)$ \\
\hline Education (years) & $15.97(2.78)$ & $16.24(2.69)$ & $16.38(2.61)$ & $16.07(2.77)$ \\
\hline APOE $₫ 4$ carriers & $471(64.08 \%)$ & 199 (59.23\%) & 165 (62.74\%) & 316 (63.07\%) \\
\hline CDR-SB & $2.07(1.90)$ & $1.86(1.91)$ & $1.40(1.43)$ & $1.77(1.75)$ \\
\hline ADAS-Cog11 & $12.54(7.22)$ & $10.55(7.06)$ & $9.53(5.40)$ & $11.31(6.86)$ \\
\hline MMSE & $26.61(2.83)$ & $26.75(3.13)$ & $27.78(2.28)$ & $27.17(2.64)$ \\
\hline ADCOMS & $0.28(0.23)$ & $0.25(0.23)$ & $0.19(0.16)$ & $0.24(0.21)$ \\
\hline PACC & $-7.46(6.00)$ & $-6.00(6.45)$ & $-4.52(4.97)$ & $-6.15(5.68)$ \\
\hline Plasma NfL (pg/mL) & 41.91 (21.18) & 44.95 (23.99) & $39.18(16.80)$ & $40.51(18.31)$ \\
\hline \multicolumn{5}{|c|}{$\begin{array}{l}\text { Data is formatted as } \mathrm{n}(\%) \text { for nominal variables and mean (standard deviation) for continuous } \\
\text { variables and scores. Samples correspond to the definition in Methods. Sample A - Longitudinal } \\
\text { analysis of cognition and baseline pNfL levels; Sample B - Longitudinal analysis of cognition and } \\
\text { ROC of pNfL; Sample C - Analysis of ROC of pNfL and imaging markers of AD; Sample D - Temporal } \\
\text { dynamics of pNfL and other markers. }\end{array}$} \\
\hline
\end{tabular}

\subsection{Association between pNfL levels and cognitive decline}

The coefficients of the interaction terms showed that increase in PNfL concentration (and baseline ROC of $\mathrm{pNfL}$ ) was significantly associated with a faster cognitive decline, as shown in Table 2. For visualisation, refer to Figure 2; we observe the same trend, implying that higher levels of baseline $\mathrm{pNfL}$, as well higher baseline ROC of pNfL, is associated with worse cognition and a faster rate of cognitive decline.

Table 2

Posterior estimates of interaction between baseline $\mathrm{pNfL}$ and time, and between baseline ROC $\mathrm{pNfL}$ and time. 


\begin{tabular}{|c|c|c|}
\hline \multirow[t]{2}{*}{ Outcome } & Baseline pNfL & Baseline ROC pNfL \\
\hline & Linear & Linear \\
\hline CDR-SB & $1.67 *(1.21,2.13)$ & $14.04^{\star}(0.97,26.12)$ \\
\hline ADAS-Cog11 & $3.46 *(2.18,4.77)$ & $50.84 *(16.42,84.82)$ \\
\hline MMSE & $-1.98 *(-2.65,-1.28)$ & $-1.44(-4.15,1.13)$ \\
\hline ADCOMS & $0.19 *(0.18,0.24)$ & $2.49(-0.01,4.94)$ \\
\hline PACC & $-2.85^{\star}(-3.86,-1.87)$ & $-2.00 *(-3.86,-0.03)$ \\
\hline \multicolumn{3}{|c|}{$\begin{array}{l}\text { * } 95 \% \text { Credible intervals do not include the null value (zero). Similar results have been obtained using } \\
\text { adjusted hierarchical beta regression model (see Supplementary Table S1). } \\
\text { The parameter estimates and } 95 \% \text { Crl for the interaction term between continuous baseline pNfL, or } \\
\text { baseline ROC pNfL, and time from baseline, from the linear regression models. All models were } \\
\text { adjusted for age at baseline (years), binary APOE status, sex, education (years), and a quadratic time } \\
\text { term. }\end{array}$} \\
\hline \multicolumn{3}{|c|}{$\begin{array}{l}\text { Note: Estimates of baseline ROC of pNfL look higher in magnitude because of the small scale of ROC } \\
\text { An increase of one unit in ROC is different to a one unit increase in the absolute value. }\end{array}$} \\
\hline
\end{tabular}

\subsection{Rate of change in pNfL levels and neuroimaging markers of $A D$}

The ROC in pNfL was significantly associated with ROC in ventricular volume $(1.23 ; 95 \% \mathrm{Crl}(0.73,1.75))$, hippocampus volume $(-1.12 ; 95 \% \mathrm{Crl}(-1.62,-0.63))$ and whole brain volume $(-0.59 ; 95 \% \mathrm{Crl}(-0.84,-0.35))$. The ROC in pNfL was not significantly associated with ROC in $\operatorname{AV} 45(0.01 ; 95 \% \operatorname{Crl}(-0.01,0.04))$. For visualisations, refer to Figure 3.

\section{4 pNfL trajectory and association with clinical progression of $A D$}

Figure 4 shows the trajectories of different markers as a function of the time from the first diagnosis of cognitive impairment as defined by a non-zero CDR-SB score, and the clinical state of individuals at each measurement after their alignment to match the population level trajectory. It is observed that, in contrast to markers that are associated with the clinical progression of $A D$, such as ADAS-Cog, MMSE and CDR-SB scores, higher $\mathrm{pNfL}$ levels do not necessarily correspond to more severe clinical states of $A D$ (for example, many $\mathrm{CN}$ individuals (blue points) may have high levels of $\mathrm{pNfL}$ ). This implies that, although pNfL can be associated with cognitive decline, pNfL levels do not reflect clearly the clinical state of 
individuals in $A D$ development and high pNfL concentration may be observed independently of the clinical diagnosis ( $\mathrm{CN}, \mathrm{MCl}, \mathrm{AD}$ dementia) of individuals on the $\mathrm{AD}$ continuum.

\section{Discussion}

This study has focused on the association between longitudinal changes in cognitive performance and continuous measures of neurofilament light measured in the plasma ( $\mathrm{pNfL}$ ) in a population at risk of developing $A D$. The rate of change of $\mathrm{pNfL}$ and associations with the rate of change of other biological markers potentially associated with the progression of $A D$ has also been investigated. We have developed novel beta regression models with random intercepts and slopes, taking into account the fact that cognitive scores are bounded, thus extending traditional linear regression models. We have also introduced the concept of 'baseline rate of change' and considered its associations with longitudinal cognitive decline. Based on the rate of change, we described the temporal dynamics of pNfL and associations with the clinical progression of $A D$, from cognitively unimpaired stages to $A D$ dementia.

We have analysed a subset of the ADNI population which is at a higher risk of developing AD in the future, on the basis of the amyloid levels at baseline. For this we have used strict dichotomous cut-offs and acknowledge that this itself comes with limitations of not being able to clearly distinguish between individuals near the cut-off level set in the analyses ${ }^{29}$. This assumption simplifies the complex realities of the dynamical behaviour of the biomarkers. Measurement of biomarker levels are inherently variable both between individuals, within individuals over time and due to measurement error, and this variability can result in classification errors.

The results of the study have demonstrated that increase in pNfL concentration (and baseline ROC of $\mathrm{pNfL}$ ) is significantly associated with cognitive performance. Higher levels of baseline $\mathrm{pNfL}$, as well as higher baseline ROC of $\mathrm{pNfL}$, are associated with faster cognitive decline. The estimates from the statistical models corresponding to these two different ways of defining of baseline $\mathrm{pNfL}$ are not directly comparable. First, because one is a sub-sample of the other and second because the scales are different. Hence, we cannot ascertain that a few longitudinal measurements of pNfL may (or may not) be a better predictor of cognitive decline than a single measurement at baseline. It should be noted that it is more difficult to see a one-unit change in ROC compared to a one-unit change in the absolute value of pNfL. Because of the high heterogeneity in pNfL measurements over time, ROC may not be the best measure. Taking the average over a short interval of time may provide more meaningful results.

The ROC in $\mathrm{pNfL}$ was significantly associated with the ROC in ventricular volume, hippocampus volume and whole brain volume, showing its value as brain damage marker. However, it was not associated with the ROC in amyloid accumulation in the brain (measured by AV-45-PET), which may be attributed to the selection criteria of the sample that has been used (amyloid positive individuals only).

Considering the trajectory of $\mathrm{pNfL}$ as a function of the time from the first non-zero CDR-SB score in relation to the clinical state of individuals ( $C N, M C l, A D$ dementia), we observed that although higher 
values of $\mathrm{pNfL}$ can predict cognitive decline, they do not necessarily correspond to more severe clinical states of AD. This may support previous studies ${ }^{6}$ which reported that NfL is not specific to AD. To some extent, it may also be due to the high degree of heterogeneity within the clinical states and the fact that the categorisation of individuals in these states was mainly based on clinical criteria ${ }^{30}$.

The study was based on one cohort deriving from the ADNI dataset. Ideally, confirmation of these trends should be derived for a number of cohort studies in different country settings to ensure that the results are not confounded by the study design ${ }^{31}$. Some of the conclusions of this study have also been recorded in previous studies ${ }^{6}$, but the present analysis employs novel methods in looking at temporal trends.

$\mathrm{NfL}$ is not a biomarker specific to AD and high levels of this marker have been associated with several other neurodegenerative disorders. However, this marker, along with other markers, could still facilitate both participant screening for clinical trials of possible $A D$ therapies and the diagnosis process ${ }^{32}$. In particular, the ability of $\mathrm{NfL}$ to predict future cognitive decline, as shown in this study, could facilitate the improvement of the criteria for participant recruitment in clinical trials. It could also be used for monitoring disease progression and response to treatment.

\section{Declarations}

\section{ACKNOWLEDGMENTS}

We thank Dr. David X Thomas (University College London) for useful discussions. SB would like to thank Clarendon Fund Scholarships at the University of Oxford for supporting the completion of this work.

This study was funded by the Medical Research Council (MRC) and Dementias Platform UK (DPUK). DPUK provided infrastructure for this project through MRC grant ref. MR/L023784/2. We acknowledge joint Centre funding from the UK Medical Research Council and Department for International Development through grant ref. MR/R015600/1.

Data collection and sharing for this project was funded by the Alzheimer's Disease Neuroimaging Initiative (ADNI) (National Institutes of Health Grant U01 AG024904) and DOD ADNI (Department of Defense award number W81XWH-12-2-0012). The ADNI was launched in 2003 as a public-private partnership, led by Principal Investigator Michael W. Weiner, MD. The primary goal of ADNI has been to test whether serial magnetic resonance imaging (MRI), positron emission tomography (PET), other biological markers, and clinical and neuropsychological assessment can be combined to measure the progression of mild cognitive impairment (MCl) and early Alzheimer's disease (AD). For up-to-date information, see www.adni-info.org.

ADNI is funded by the National Institute on Aging, the National Institute of Biomedical Imaging and Bioengineering, and through generous contributions from the following: AbbVie, Alzheimer's Association; 
Alzheimer's Drug Discovery Foundation; Araclon Biotech; BioClinica, Inc.; Biogen; Bristol-Myers Squibb Company; CereSpir, Inc.; Cogstate; Eisai Inc.; Elan Pharmaceuticals, Inc.; Eli Lilly and Company; Eurolmmun; F. Hoffmann-La Roche Ltd and its affiliated company Genentech, Inc.; Fujirebio; GE Healthcare; IXICO Ltd.; Janssen Alzheimer Immunotherapy Research \& Development, LLC.; Johnson \& Johnson Pharmaceutical Research \& Development LLC.; Lumosity; Lundbeck; Merck \& Co., Inc.; Meso Scale Diagnostics, LLC.; NeuroRx Research; Neurotrack Technologies; Novartis Pharmaceuticals Corporation; Pfizer Inc.; Piramal Imaging; Servier; Takeda Pharmaceutical Company; and Transition Therapeutics. The Canadian Institutes of Health Research is providing funds to support ADNI clinical sites in Canada. Private sector contributions are facilitated by the Foundation for the National Institutes of Health (www.fnih.org). The grantee organization is the Northern California Institute for Research and Education, and the study is coordinated by the Alzheimer's Therapeutic Research Institute at the University of Southern California. ADNI data are disseminated by the Laboratory for Neuro Imaging at the University of Southern California.

\section{COMPETING INTERESTS}

S Bajaj, C Hadjichrysanthou, K McRae-McKee, F.D Wolf, RM Anderson report no conflicts of interest related to the article.

\section{DATA AVAILABILITY}

The datasets generated and analysed during the current study are available in the ADNI data repository, http://adni.loni.usc.edu/data-samples/access-data/.

\section{ETHICS DECLARATION}

The study procedures were approved by the institutional review boards of all participating centers (https://adni.loni.usc.edu/wp-content/uploads/how_to_apply/ADNI_Acknowledgement_List.pdf), and written informed consent was obtained from all participants or their authorized representatives. Ethics approval was obtained from the institutional review boards of each institution involved: Oregon Health and Science University; University of Southern California; University of California-San Diego; University of Michigan; Mayo Clinic, Rochester; Baylor College of Medicine; Columbia University Medical Center; Washington University, St. Louis; University of Alabama at Birmingham; Mount Sinai School of Medicine; Rush University Medical Center; Wien Center; Johns Hopkins University; New York University; Duke University Medical Center; University of Pennsylvania; University of Kentucky; University of Pittsburgh; University of Rochester Medical Center; University of California, Irvine; University of Texas Southwestern Medical School; Emory University; University of Kansas, Medical Center; University of California, Los Angeles; Mayo Clinic, Jacksonville; Indiana University; Yale University School of Medicine; McGill University, Montreal-Jewish General Hospital; Sunnybrook Health Sciences, Ontario; U.B.C. Clinic for AD \& 
Related Disorders; Cognitive Neurology-St. Joseph's, Ontario; Cleveland Clinic Lou Ruvo Center for Brain Health; Northwestern University; Premiere Research Inst (Palm Beach Neurology); Georgetown University Medical Center; Brigham and Women's Hospital; Stanford University; Banner Sun Health Research Institute; Boston University; Howard University; Case Western Reserve University; University of California, Davis-Sacramento; Neurological Care of CNY; Parkwood Hospital; University of Wisconsin; University of California, Irvine-BIC; Banner Alzheimer's Institute; Dent Neurologic Institute; Ohio State University; Albany Medical College; Hartford Hospital, Olin Neuropsychiatry Research Center; Dartmouth-Hitchcock Medical Center; Wake Forest University Health Sciences; Rhode Island Hospital; Butler Hospital; UC San Francisco; Medical University South Carolina; St. Joseph's Health Care Nathan Kline Institute; University of lowa College of Medicine; and Cornell University and University of South Florida: USF Health Byrd Alzheimer's Institute. The investigators within the ADNI contributed to the design and implementation of the $A D N I$ and/or provided data but did not participate in the analysis or writing of this report. A complete listing of $A D N I$ investigators can be found online

(http://adni.loni.usc.edu/wp-content/uploads/how_to_apply/ADNI_Acknowledgement_List.pdf).

\section{FUNDING}

This study was funded by the Medical Research Council (MRC) and Dementias Platform UK (DPUK). The funding body had no role in the design of the study and collection, analysis, and interpretation of data and in writing the manuscript.

\section{References}

1. Khachaturian, Z. S. Diagnosis of Alzheimer's Disease. Arch. Neurol. 42, 1097-1105 (1985).

2. Jack, C. R. et al. NIA-AA Research Framework: Toward a biological definition of Alzheimer's disease. Alzheimer's Dement. 14, 535-562 (2018).

3. Hadjichrysanthou, C. et al. The dynamics of biomarkers across the clinical spectrum of Alzheimer's disease. Alzheimers. Res. Ther. 12, 74 (2020).

4. Fossati, S. et al. Plasma tau complements CSF tau and P-tau in the diagnosis of Alzheimer's disease. Alzheimer's Dement. (Amsterdam, Netherlands) 11, 483-492 (2019).

5. Nakamura, A. et al. High performance plasma amyloid- $\beta$ biomarkers for Alzheimer's disease. Nature 554, 249-254 (2018).

6. Mattsson, N., Andreasson, U., Zetterberg, H., Blennow, K. \& Initiative, for the A. D. N. Association of Plasma Neurofilament Light With Neurodegeneration in Patients With Alzheimer Disease. JAMA Neurol. 74, 557-566 (2017).

7. Mattsson, N., Cullen, N. C., Andreasson, U., Zetterberg, H. \& Blennow, K. Association Between Longitudinal Plasma Neurofilament Light and Neurodegeneration in Patients With Alzheimer Disease. JAMA Neurol. 76, 791-799 (2019). 
8. Gaetani, L. et al. Neurofilament light chain as a biomarker in neurological disorders. J. Neurol. Neurosurg. Psychiatry 90, 870-881 (2019).

9. Lu, C.-H. et al. Neurofilament light chain. Neurology 84, 2247 LP - 2257 (2015).

10. Disanto, G. et al. Serum Neurofilament light: A biomarker of neuronal damage in multiple sclerosis. Ann. Neurol. 81, 857-870 (2017).

11. Kern, S. et al. Association of Cerebrospinal Fluid Neurofilament Light Protein With Risk of Mild Cognitive Impairment Among Individuals Without Cognitive Impairment. JAMA Neurol. 76, 187-193 (2019).

12. de Wolf, F. et al. Plasma tau, neurofilament light chain and amyloid- $\beta$ levels and risk of dementia; a population-based cohort study. Brain 143, 1220-1232 (2020).

13. Landau, S. M. et al. Amyloid deposition, hypometabolism, and longitudinal cognitive decline. Ann. Neurol. 72, 578-586 (2012).

14. Hansson, 0 . et al. CSF biomarkers of Alzheimer's disease concord with amyloid- $\beta$ PET and predict clinical progression: A study of fully automated immunoassays in BioFINDER and ADNI cohorts. Alzheimers. Dement. 14, 1470-1481 (2018).

15. Folstein, M. F., Folstein, S. E. \& McHugh, P. R. "Mini-mental state”: A practical method for grading the cognitive state of patients for the clinician. J. Psychiatr. Res. 12, 189-198 (1975).

16. Mohs, R. C. et al. Development of cognitive instruments for use in clinical trials of antidementia drugs: additions to the Alzheimer's Disease Assessment Scale that broaden its scope. The Alzheimer's Disease Cooperative Study. Alzheimer Dis. Assoc. Disord. 11 Suppl 2, S13-21 (1997).

17. Hughes, C. P., Berg, L., Danziger, W. L., Coben, L. A. \& Martin, R. L. A new clinical scale for the staging of dementia. Br. J. Psychiatry 140, 566-572 (1982).

18. Berg, L. et al. Mild senile dementia of the alzheimer type: 2. Longitudinal assessment. Ann. Neurol. 23, 477-484 (1988).

19. Wang, J. et al. ADCOMS: a composite clinical outcome for prodromal Alzheimer\&\#039;s disease trials. J. Neurol. Neurosurg. \&amp;amp; Psychiatry 87, 993 LP - 999 (2016).

20. Donohue, M. C. et al. The preclinical Alzheimer cognitive composite: measuring amyloid-related decline. JAMA Neurol. 71, 961-970 (2014).

21. Donohue, M. C. et al. Association Between Elevated Brain Amyloid and Subsequent Cognitive Decline Among Cognitively Normal Persons. JAMA 317, 2305-2316 (2017).

22. Fox, N. C. \& Schott, J. M. Imaging cerebral atrophy: normal ageing to Alzheimer's disease. Lancet (London, England) 363, 392-394 (2004).

23. Clark, C. M. et al. Use of florbetapir-PET for imaging beta-amyloid pathology. JAMA 305, 275-283 (2011).

24. Whitwell, J. L., Crum, W. R., Watt, H. C. \& Fox, N. C. Normalization of cerebral volumes by use of intracranial volume: implications for longitudinal quantitative MR imaging. AJNR. Am. J. Neuroradiol. 22, 1483-1489 (2001). 
25. Thomas, D. X. et al. Association of TDP-43 proteinopathy, cerebral amyloid angiopathy, and Lewy bodies with cognitive impairment in individuals with or without Alzheimer's disease neuropathology. Sci. Rep. 10, 14579 (2020).

26. Villemagne, V. L. et al. Amyloid \&\#x3b2; deposition, neurodegeneration, and cognitive decline in sporadic Alzheimer's disease: a prospective cohort study. Lancet Neurol. 12, 357-367 (2013).

27. Roe, C. M. et al. Incident cognitive impairment: longitudinal changes in molecular, structural and cognitive biomarkers. Brain 141, 3233-3248 (2018).

28. Carpenter, B. et al. Stan: A Probabilistic Programming Language. J. Stat. Software; Vol 1, Issue 1 (2017) doi:10.18637/jss.v076.i01.

29. McRae-McKee, K. et al. Perspective: Clinical relevance of the dichotomous classification of Alzheimer's disease biomarkers: Should there be a \&\#x201c;gray zone\&\#x201d;? Alzheimer's Dement. J. Alzheimer's Assoc. (2019) doi:10.1016/j.jalz.2019.07.010.

30. Hadjichrysanthou, C. et al. Potential Factors Associated with Cognitive Improvement of Individuals Diagnosed with Mild Cognitive Impairment or Dementia in Longitudinal Studies. J. Alzheimer's Dis. 66, 587-600 (2018).

31. Evans, S. et al. Alzheimer's disease progression and risk factors: A standardized comparison between six large data sets. Alzheimer's Dement. (New York, N. Y.) 5, 515-523 (2019).

32. Anderson, R. M., Hadjichrysanthou, C., Evans, S. \& Wong, M. M. Why do so many clinical trials of therapies for Alzheimer's disease fail? Lancet (London, England) 390, 2327-2329 (2017).

\section{Figures}




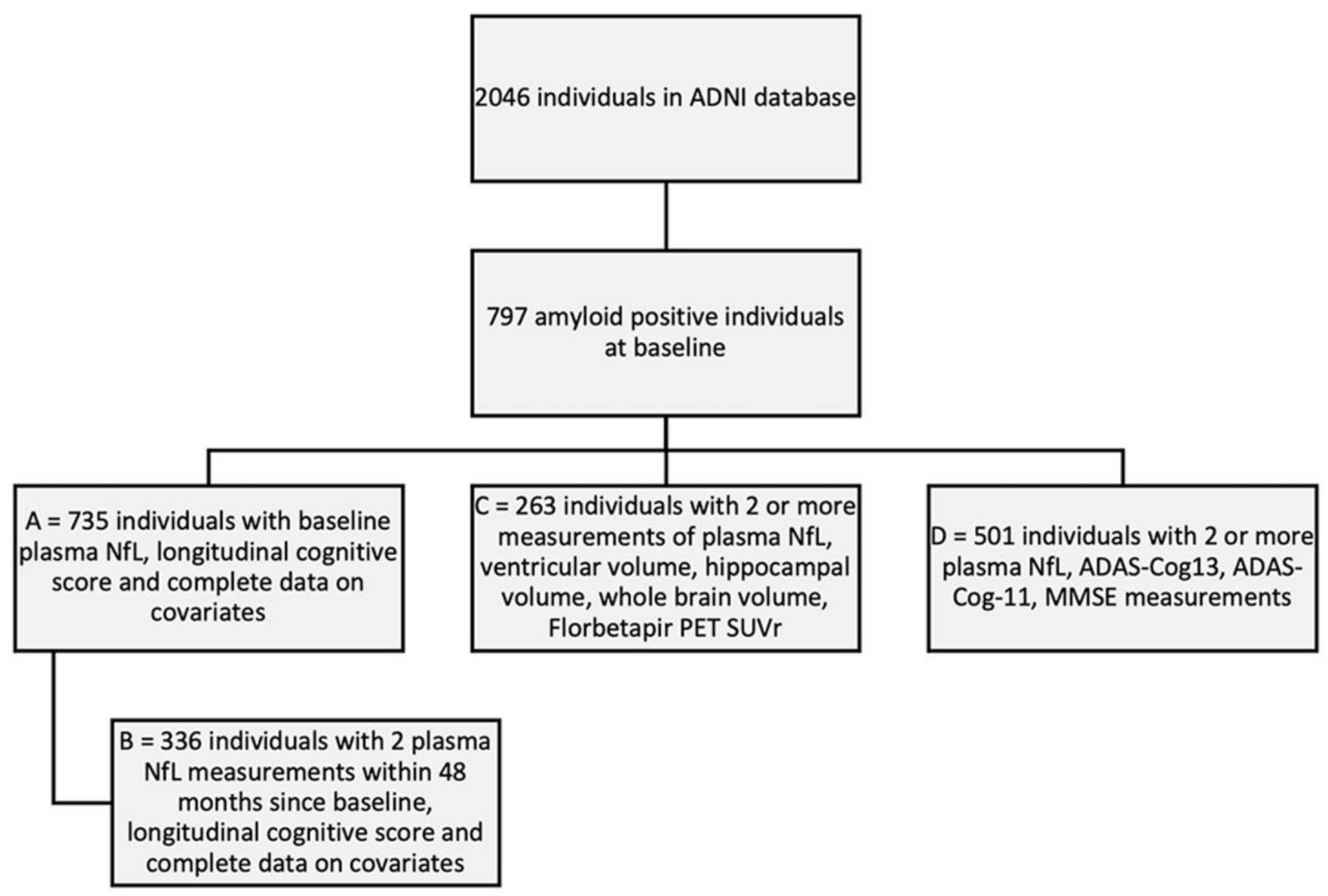

\section{Figure 1}

Sample selection 

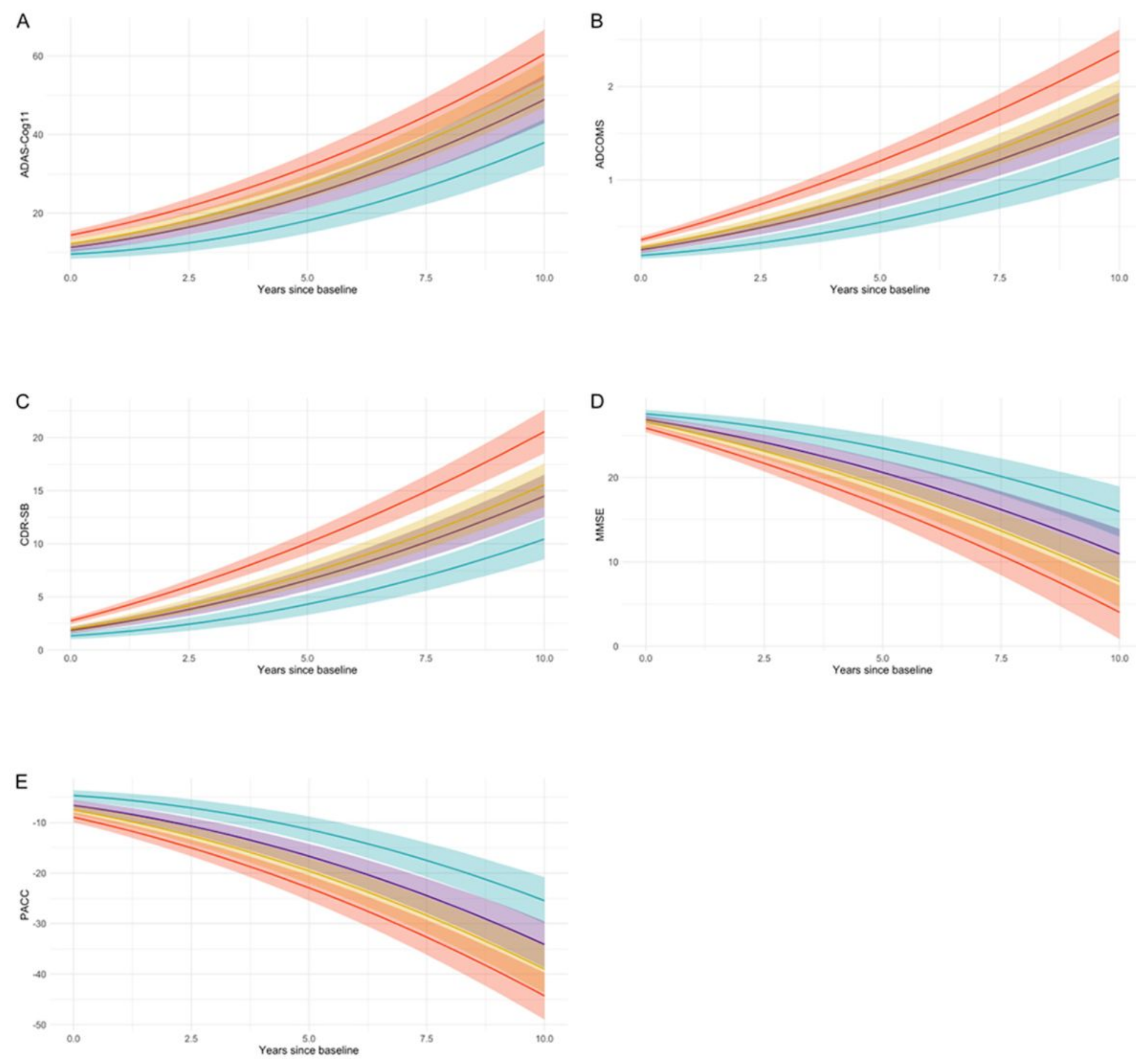

\section{Figure 2}

Association between baseline $\mathrm{pNfL}$ and longitudinal cognitive scores. The solid line and shaded area represent the estimated mean and $95 \%$ credible intervals of cognitive scores, respectively, for the quartiles of baseline pNfL. The blue, purple, yellow and orange curves correspond to $1 \mathrm{st}, 2 \mathrm{nd}, 3 \mathrm{rd}$ and 4 th quartile respectively. Plots were produced using adjusted hierarchical linear regression for the following values for the confounders: age at baseline (years) $=73.78$, APOE positive status, female, education (years) $=15.97$. A quadratic time term for the linear regression model was used. CDR-SB = Clinical Dementia Rating-Sum 
of Boxes, ADAS-Cog11 = Alzheimer's Disease Assessment Scale-Cognitive 11, MMSE = Mini-Mental State Examination, $\mathrm{ADCOMS}=$ Alzheimer's Disease Composite Score, $\mathrm{PACC}=$ Preclinical Alzheimer's Cognitive Composite including Trail-Making Test B. Similar results have been obtained using adjusted hierarchical beta regression model (see Supplementary Fig. S1).
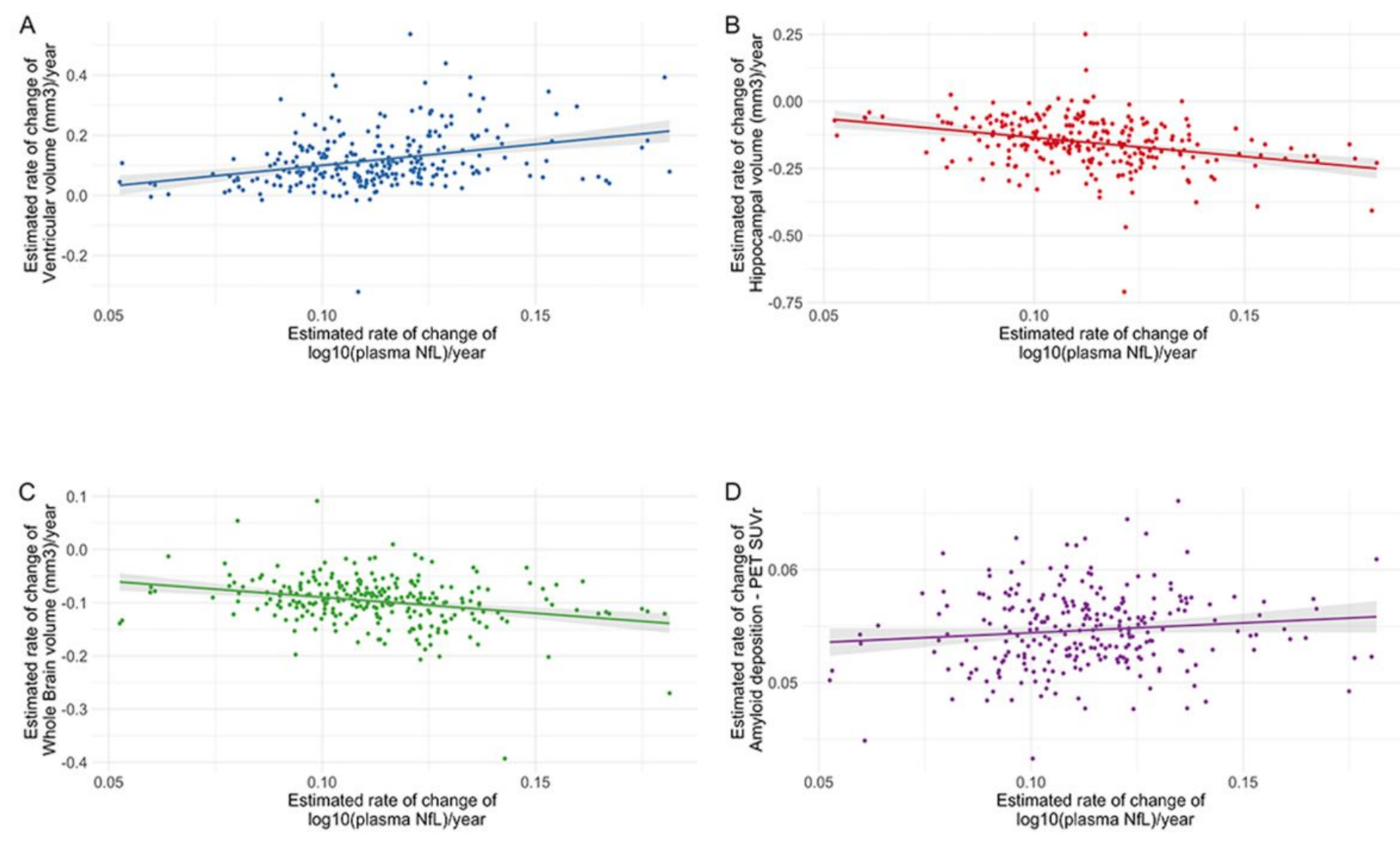

Figure 3

Association between ROC of pNfL and ROC of other neuroimaging markers. Linear regression between estimated rate of change in $\mathrm{pNfL}$ and estimated rate of change in ventricular volume, hippocampal 
volume, whole brain volume and amyloid accumulation (Standard Uptake Value Ratio SUVR) using Florbetapir in PET scans.
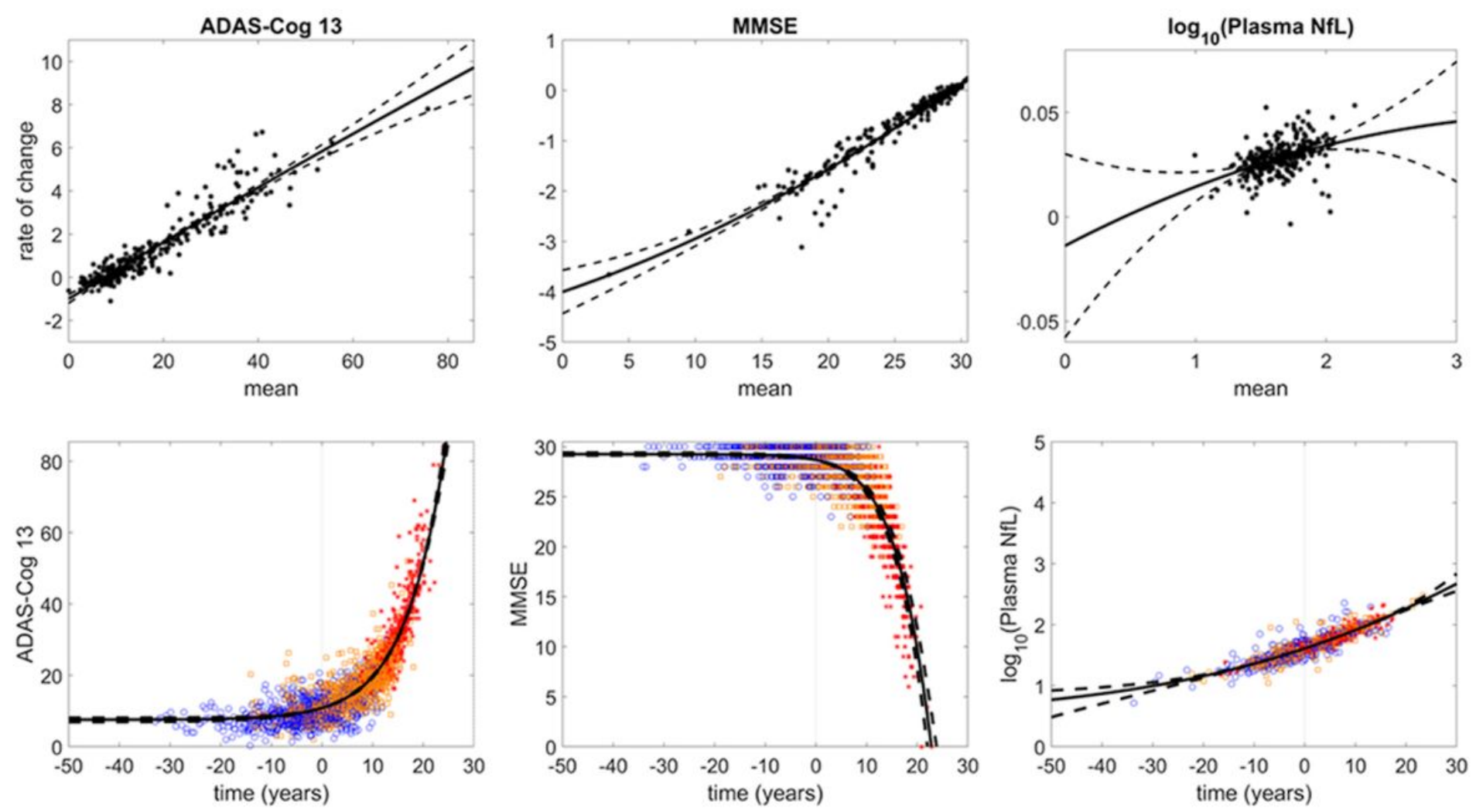

\section{Figure 4}

Temporal dynamics of biomarkers before and after CDR-SB > 0 onset. Top row: the rate of change as a function of the mean for ADAS-Cog 13, MMSE, and $\log 10(\mathrm{pNfL})$. Bottom row: The average trajectory of the above scores as a function of time from the first non-zero CDR-SB value (time $=0$ ). The individual 
data have been aligned to match the population-level trajectory - Blue: $\mathrm{CN}$, Orange: $\mathrm{MCl}, \mathrm{Red}: \mathrm{AD}$. The dashed lines represent the $95 \%$ confidence interval for the mean.

\section{Supplementary Files}

This is a list of supplementary files associated with this preprint. Click to download.

- ADNInflSupplementaryMaterial.docx 